\title{
On monotone multivalued transformations
}

\author{
Buthinah A. Bin Dehaisha, Mohamed A. Khamsib,c,* \\ ${ }^{a}$ Department of Mathematics, Faculty of Science, King Abdulaziz University, Jeddah, Saudi Arabia. \\ ${ }^{b}$ Department of Mathematics \& Statistics, King Fahd University of Petroleum and Minerals Dhahran 31261, Saudi Arabia. \\ ${ }^{c}$ Department of Mathematical Sciences, University of Texas at El Paso, El Paso, TX 79968, U.S.A.
}

Communicated by Y. J. Cho

\begin{abstract}
In this work, we discuss the recently introduced monotone $\tau$-Opial condition in Banach spaces which admit a sequence of monotone approximations of the identity. Then we give a fixed point theorem for monotone multivalued nonexpansive mappings in Banach spaces satisfying the monotone $\tau$-Opial condition. This result generalizes those of Markin, Browder and Lami Dozo to monotone mappings. (C)2017 All rights reserved.
\end{abstract}

Keywords: Fixed point, systems of projections, monotone Opial condition, monotone nonexpansive mappings, multivalued mappings.

2010 MSC: 47H10, 46B99.

\section{Introduction}

The extension of many fixed point theorems of singlevalued mappings to the multivalued case was never an easy one. Recall that the first attempts was carried by Markin [16] in Hilbert spaces, by Browder [5] in spaces having a weakly continuous duality mapping, and by Lami Dozo [14] in spaces satisfying the weak Opial condition. For a nice survey on multivalued mappings, we recommend the paper by Benavides and Ramírez [4].

The study of fixed points of monotone mappings attracted some attention following the extension of the Banach Contraction Principle [3] by Ran and Reurings [18] in partially ordered metric spaces. For an extensive list of references and historical facts about monotone mappings, we recommend the survey by Bachar and Khamsi [2]. In this work, we extend the main ideas of Lami Dozo [14] to the case of monotone multivalued mappings. In particular, we introduce the concept of monotone approximation of the identity of a Banach space.

For more on the metric fixed point theory, we recommend the book by Khamsi and Kirk [12].

\footnotetext{
*Corresponding author

Email addresses: bbindehaish@yahoo.com, bbendehaish@kau.edu.sa (Buthinah A. Bin Dehaish), mohamed@utep.edu, mkhamsi@kfupm.edu.sa (Mohamed A. Khamsi)
}

doi:10.22436/jnsa.010.10.18 


\section{Monotone Opial condition}

Throughout, $(X,\|\|,. \preceq)$ stands for a partially ordered Banach space such that $x \preceq y$ implies

$$
\alpha x+z \preceq \alpha y+z
$$

for any $x, y, z \in X$ and $\alpha \in[0,+\infty)$. We will assume that order intervals are closed. Recall that an order interval is any of the sets

$$
[x, \rightarrow)=\{y \in X ; x \preceq y\} \text { and }(\leftarrow, x]=\{y \in X ; y \preceq x\}
$$

for any $x \in X$. Let $\tau$ be a topology on $X$ for which order intervals are $\tau$-closed. Let us next recall the definition of $\tau$-Opial condition $[6,17]$.

Definition 2.1. We will say that $X$ satisfies the $\tau$-Opial condition if for any sequence $\left\{x_{n}\right\}$ in $X$ which $\tau$-converges to $x$, we have

$$
\limsup _{n \rightarrow+\infty}\left\|x_{n}-x\right\|<\limsup _{n \rightarrow+\infty}\left\|x_{n}-y\right\|
$$

for any $y \in X$ such that $y \neq x$.

Very early on, this property played a major role in the study of the fixed point property of nonexpansive mappings [6]. For example, Opial [17] noted that the classical Banach spaces $\ell^{p}$ enjoyed the weak-Opial condition while $\mathrm{L}^{p}([0,1])$ fails it for $p \neq 2$ and $1<p<\infty$. In recent study of the fixed point property of monotone nonexpansive mappings, the authors introduced the concept of monotone Opial condition as follows.

Definition 2.2 ([1]). We will say that $X$ satisfies the monotone $\tau$-Opial condition if for any monotone increasing (resp. decreasing) sequence $\left\{x_{n}\right\}$ in $X$ which $\tau$-converges to $x$, we have

$$
\limsup _{n \rightarrow+\infty}\left\|x_{n}-x\right\|<\limsup _{n \rightarrow+\infty}\left\|x_{n}-y\right\|
$$

for any $y \in \bigcap_{n \geqslant 1}\left[x_{n}, \rightarrow\right)$ (resp. $\left.y \in \bigcap_{n \geqslant 1}\left(\leftarrow, x_{n}\right]\right)$ such that $y \neq x$.

Note that since we assumed that order intervals are $\tau$-closed, then we have $y \in \bigcap_{n \geqslant 1}\left[x_{n}, \rightarrow\right)$ if and only if $x \preceq y$ (resp. $y \in \bigcap_{n \geqslant 1}\left(\leftarrow, x_{n}\right]$ if and only if $y \preceq x$ ). In [1], it is proved that any lattice Banach space with a monotone norm satisfies a large monotone weak-Opial condition. But if the norm is uniformly convex, then we have the monotone weak-Opial condition. This is amazing because it allows one to prove that $\mathrm{L}^{p}([0,1])$ satisfies the monotone weak-Opial condition, for $p \neq 2$ and $1<p<\infty$.

Next, we extend the concept of the approximation of the identity as introduced by Lami Dozo [14] to the monotone case.

Definition 2.3. A sequence of bounded linear operators $\left\{P_{n}\right\}_{n} \geqslant 1$ of $X$ is said to be a monotone $\tau$-approximation of the identity, if

(1) $\lim _{n \rightarrow \infty}\left\|x-P_{n}(x)\right\|=0$, for any $x \in X$;

(2) $\lim _{n \rightarrow \infty}\left\|P_{i}\left(x_{n}\right)\right\|=0$, for any $i \geqslant 1$ and any sequence $\left\{x_{n}\right\}$ which $\tau$-converges to 0 ;

(3) $P_{n}$ and $Q_{n}=I-P_{n}$ are monotone operators, where $I: X \rightarrow X$ is the identity mapping.

We will say that $\left\{P_{n}\right\}$ satisfies the property (L) if there exists a continuous function $\delta:[0, \infty) \times[0, \infty) \rightarrow$ $[0, \infty)$ such that

$$
\left\|P_{n}(u)+Q_{n}(v)\right\| \geqslant \delta\left(\left\|P_{n}(u)\right\|,\left\|Q_{n}(v)\right\|\right)
$$

for any $u, v \in X$ such that $u \succeq 0$ and $v \succeq 0$ and $n \geqslant 1$. Moreover the function $\delta$ satisfies $\delta(0, r)=\delta(r, 0)=r$, $\delta(r, s)>r$ and $\delta(s, r)>r$ for any $r \geqslant 0$ and $s>0$. 
The origin of the property (L) may be found in [11] where this property was introduced and investigated in relation with the Opial and Kadec-Klee properties. The following example will help shed some interesting properties about this new concept. It is inspired from the original work of Lim [15].

Example 2.4. Consider the Banach space $\ell^{p}$, with $1<p<\infty$. For any $x=\left(x_{n}\right) \in \ell^{p}$, define

$$
x^{+}=\left(\max \left(x_{n}, 0\right)\right) \text { and } x^{-}=\left(-\min \left(x_{n}, 0\right)\right) .
$$

Define the new norm in $\ell^{p}$ defined by

$$
\|x\|=\left\|x^{+}\right\|_{p}+\left\|x^{-}\right\|_{p}
$$

for any $x \in \ell^{p}$. It is easy to check that $\|$.$\| is an equivalent norm to \|\cdot\|_{p}$. Next consider the sequence of natural projections $\left\{P_{n}\right\}$ associated to the canonical Schauder basis of $\ell^{p}$, i.e.

$$
P_{n}(x)=\left(x_{1}, x_{2}, \cdots, x_{n}, 0,0,0, \cdots\right), \quad x \in \ell^{p} .
$$

Then $\left\{Q_{n}\right\}$ are also projections defined by

$$
\mathrm{Q}_{n}(x)=\left(0,0, \cdots, x_{n+1}, x_{n+2}, \cdots\right), \quad x \in \ell^{p} .
$$

Note that if $u, v \in \ell^{p}$ are such that $u \succeq 0$ and $v \succeq 0$, then we have $u=u^{+}$and $v=v^{+}$and

$$
\left\|P_{n}(u)+Q_{n}(v)\right\|=\left\|P_{n}(u)+Q_{n}(v)\right\|_{p}=\left(\left\|P_{n}(u)\right\|_{p}^{p}+\left\|Q_{n}(v)\right\|_{p}^{p}\right)^{1 / p}
$$

which implies

$$
\left\|P_{n}(u)+Q_{n}(v)\right\|=\left(\left\|P_{n}(u)\right\|^{p}+\left\|Q_{n}(v)\right\|^{p}\right)^{1 / p}
$$

for any $n \geqslant 1$. If we take $\delta(r, s)=\left(r^{p}+s^{p}\right)^{1 / p}$, the sequence $\left\{P_{n}\right\}$ satisfies the property $(L)$. And if we take $\tau$ to be the weak topology, then $\left\{\mathrm{P}_{\mathrm{n}}\right\}$ is a monotone weak-approximation of the identity. Note that $\left(\ell^{p},\|\|.\right)$ is not strictly convex since

$$
\left\|e_{1}\right\|=\left\|-e_{2}\right\|=\left\|\left(e_{1}-e_{2}\right) / 2\right\|=1,
$$

where $e_{1}=(1,0,0, \cdots)$ and $e_{2}=(0,1,0,0, \cdots)$.

The following result may be seen as an analogue to [14, Theorem 2.1].

Theorem 2.5. Let $(X,\|\|,. \preceq)$ and $\tau$ be as described before. Assume $X$ has a monotone $\tau$-approximation of the identity $\left\{\mathrm{P}_{\mathrm{n}}\right\}$ which satisfies the property $(\mathrm{L})$. Then $\mathrm{X}$ satisfies the monotone $\tau$-Opial condition.

Proof. Let $\left\{x_{n}\right\}$ be a monotone sequence in $X$ which $\tau$-converges to $x$. Without loss of generality, we assume $\left\{x_{n}\right\}$ is monotone increasing. Since order intervals are $\tau$-closed, we know that $x_{n} \preceq x$, for any $n \geqslant 1$. Let $y \neq x$ and $x \preceq y$. We have

$$
y-x_{n}=y-x+\left(x-x_{n}\right), \quad n \geqslant 1 .
$$

Set $u=y-x$ and $u_{n}=x-x_{n}$, for $n \geqslant 1$. Using the properties satisfied by the order $\preceq$ and the topology $\tau$, we conclude that $u \succeq 0, u_{n} \succeq 0$, for any $n \geqslant 1, u \neq 0$, and $\left\{u_{n}\right\} \tau$-converges to 0 . Since $P_{i}+Q_{i}=I$, we get

$$
u+u_{n}=P_{i}(u)+Q_{i}\left(u_{n}\right)+Q_{i}(u)+P_{i}\left(u_{n}\right)
$$

for any $n, i \geqslant 1$. Since $\left\{P_{n}\right\}$ is a monotone $\tau$-approximation of the identity which satisfies the (L) property, we have

$$
\lim _{i \rightarrow \infty}\left\|Q_{i}(u)\right\|=\lim _{n \rightarrow \infty}\left\|P_{i}\left(u_{n}\right)\right\|=0 .
$$


Hence

$$
\begin{aligned}
\left\|u+u_{n}\right\| & \geqslant\left\|P_{i}(u)+Q_{i}\left(u_{n}\right)\right\|-\left\|Q_{i}(u)\right\|-\left\|P_{i}\left(u_{n}\right)\right\| \\
& \geqslant \delta\left(\left\|P_{i}(u)\right\|,\left\|Q_{i}\left(u_{n}\right)\right\|\right)-\left\|Q_{i}(u)\right\|-\left\|P_{\mathfrak{i}}\left(u_{n}\right)\right\|
\end{aligned}
$$

for any $n, i \geqslant 1$. If we let $n \rightarrow \infty$, we get

$$
\limsup _{n \rightarrow \infty}\left\|u+u_{n}\right\| \geqslant \delta\left(\left\|P_{i}(u)\right\|, \limsup _{n \rightarrow \infty}\left\|u_{n}\right\|\right)-\left\|Q_{i}(u)\right\|,
$$

since $\limsup _{n \rightarrow \infty}\left\|Q_{i}\left(u_{n}\right)\right\|=\limsup _{n \rightarrow \infty}\left\|u_{n}\right\|$, for any $i \geqslant 1$. Next, we let $i \rightarrow \infty$, we get

$$
\limsup _{n \rightarrow \infty}\left\|u+u_{n}\right\| \geqslant \delta\left(\|u\|, \limsup _{n \rightarrow \infty}\left\|u_{n}\right\|\right),
$$

since $\limsup \left\|P_{i}(u)\right\|=\|\mathfrak{u}\|$. Since $\|u\|>0$ we obtain

$$
\limsup _{n \rightarrow \infty}\left\|u+u_{n}\right\|>\limsup _{n \rightarrow \infty}\left\|u_{n}\right\|
$$

i.e.,

$$
\limsup _{n \rightarrow \infty}\left\|x_{n}-y\right\|>\limsup _{n \rightarrow \infty}\left\|x_{n}-x\right\| .
$$

The proof of our claim is complete.

As a corollary, we have the following result.

Corollary 2.6. Let $\left(\ell^{p},\|\cdot\|\right)$, with $1<p<\infty$, the Banach space described in Example 2.4. Then $\left(\ell^{p},\|\cdot\|\right)$ satisfies the monotone weak-Opial condition.

This is truly amazing since $\left(\ell^{p},\|\cdot\|\right)$ fails to be uniformly convex. In the next section, we give a fixed point result for multivalued monotone nonexpansive mappings in Banach spaces which satisfy the monotone Opial condition.

\section{Fixed points of monotone multivalued nonexpansive mappings}

Before we give the definition of monotone multivalued nonexpansive mappings, we need the following definition.

Definition 3.1. Let $(X,\|\|,. \preceq)$ be a Banach space endowed with a partial order. Let $C$ be a nonempty subset of $X$. Let $T: C \rightarrow C$ be a map.

(a) $\mathrm{T}$ is said to be monotone if

$$
x \preceq y \Longrightarrow T(x) \preceq T(y)
$$

for any $x, y \in M$.

(b) $\mathrm{T}$ is said to be monotone Lipschitzian if $\mathrm{T}$ is monotone and there exists $k \geqslant 0$ such that

$$
\|T(x)-T(y)\| \leqslant k\|x-y\|
$$

for any $x, y \in C$ such that $x \preceq y$.

If $k<1$, then we say that $T$ is a monotone contraction mapping. If $k=1, T$ is called a monotone nonexpansive mapping. A point $x \in C$ is said to be a fixed point of $T$ whenever $T(x)=x$.

Note that classical definitions of nonexpansive multivalued mappings use the Hausdorff distance. This will force the multivalued mappings to have closed and bounded values. Next, we define the concept of monotone nonexpansive multivalued mappings defined on a partially ordered Banach space which, in single-valued case, coincides with the definition of monotone nonexpansive mappings. 
Definition 3.2. Let $(X,\|\cdot\|, \preceq)$ be a Banach space endowed with a partial order and $C$ a nonempty subset of $X$. A multivalued mapping $\mathrm{T}: \mathrm{C} \rightarrow 2^{\mathrm{C}}$ with nonempty values is said to be monotone nonexpansive if for any $x, y \in C$ with $x \preceq y$ and any $u \in T(x)$ there exists $v \in T(y)$ such that

$$
u \preceq v \text { and }\|u-v\| \leqslant\|x-y\| .
$$

A point $x$ is said to be a fixed point of $T$ if and only if $x \in T(x)$.

As we said before, monotone Lipschitzian mappings are not necessarily continuous. They usually have good topological behaviors on comparable elements. In order to take advantage of this point, one approach to study such mappings is to use iterative methods. Let $(X,\|\cdot\|, \preceq)$ and $\tau$ be as described before. Mostly we will consider sequential convergence with respect to $\tau$. A nice discussion about the use of a topology versus a sequential convergence may be found in the work of Dudley [7].

Let $C$ be a convex nonempty subset of a partially ordered Banach space $X$ not reduced to one point. Let $\mathrm{T}: \mathrm{C} \rightarrow 2^{\mathrm{C}}$ be a monotone multivalued nonexpansive mapping with nonempty values. Set

$$
\mathrm{C}_{\mathrm{T}}=\{x \in \mathrm{C} ; \mathrm{x} \preceq \mathrm{y} \text { or } \mathrm{y} \preceq x \text { for some } \mathrm{y} \in \mathrm{T}(\mathrm{x})\} .
$$

Assume that $C_{T}$ is not empty. Fix $\lambda \in(0,1)$ and $x_{0} \in C_{T}$. Without loss of generality, assume there exists $y_{0} \in T\left(x_{0}\right)$ such that $x_{0} \preceq y_{0}$. Then $x_{1}=\lambda x_{0}+(1-\lambda) y_{0} \in C$. Since order intervals are convex, we have $x_{0} \preceq x_{1} \preceq y_{0}$. Since $T$ is monotone multivalued nonexpansive, then there exists $y_{1} \in T\left(x_{1}\right)$ such that $y_{0} \preceq y_{1}$ and $\left\|y_{0}-y_{1}\right\| \leqslant\left\|x_{0}-x_{1}\right\|$. We have $x_{0} \preceq x_{1} \preceq y_{0} \preceq y_{1}$. Set $x_{2}=\lambda x_{1}+(1-\lambda) y_{1}$. Then $x_{2} \in C$ and $x_{1} \preceq x_{2} \preceq y_{1}$ since order intervals are convex. Since $T$ is monotone multivalued nonexpansive, then there exists $y_{2} \in T\left(x_{2}\right)$ such that $y_{1} \preceq y_{2}$ and $\left\|y_{1}-y_{2}\right\| \leqslant\left\|x_{1}-x_{2}\right\|$. We have $x_{1} \preceq x_{2} \preceq y_{1} \preceq y_{2}$. By induction, we build two sequences $\left\{x_{n}\right\}$ and $\left\{y_{n}\right\}$ in $C$ defined by what is known as the Krasnoselskii-Ishikawa $[10,13]$ iteration

$$
x_{n+1}=\lambda x_{n}+(1-\lambda) y_{n}, n \geqslant 1,
$$

such that $\left\|y_{n}-y_{n+1}\right\| \leqslant\left\|x_{n}-x_{n+1}\right\|, y_{n} \in T\left(x_{n}\right)$ and $x_{n} \preceq x_{n+1} \preceq y_{n} \preceq y_{n+1}$, for any $n \in \mathbb{N}$.

In order to proceed further, we will need the following fundamental property satisfied by $\left\{x_{n}\right\}$.

Proposition $3.3([8,9])$. Consider the sequences $\left\{x_{n}\right\}$ and $\left\{y_{n}\right\}$ generated by $\lambda, x_{0}$ and the iteration (3.1). Then the following inequality holds

(GK) $(1+n \lambda)\left\|y_{i}-x_{i}\right\| \leqslant\left\|y_{i+n}-x_{i}\right\|+(1-\lambda)^{-n}\left(\left\|y_{i}-x_{i}\right\|-\left\|y_{i+n}-x_{i+n}\right\|\right), \forall i, n \in \mathbb{N}$.

If $\mathrm{C}$ is assumed to be bounded, then we have $\lim _{n \rightarrow+\infty}\left\|x_{n}-y_{n}\right\|=0$, i.e., $\left\{x_{n}\right\}$ is an approximate fixed point sequence of $\mathrm{T}$.

The following result will be helpful to prove the main fixed point theorem of this work.

Lemma 3.4. Assume that $\mathrm{C}$ is a convex and bounded nonempty subset of $\mathrm{X}$ not reduced to one point. Let $\mathrm{T}$ : $\mathrm{C} \rightarrow 2^{\mathrm{C}}$ be a monotone nonexpansive multivalued mapping with nonempty values. Assume $\mathrm{C}_{\mathrm{T}}$ is not empty. Fix $\lambda \in(0,1)$ and $x_{0} \in C_{T}$. Consider the sequences $\left\{x_{n}\right\}$ and $\left\{y_{n}\right\}$ in $C$ generated by $\lambda, x_{0}$ and the iteration (3.1). Then $\left\{x_{n}\right\}$ has at most one $\tau$-cluster point.

Proof. Since $x_{0} \in C_{T}$, there exists $y_{0} \in T\left(x_{0}\right)$ which is comparable to $x_{0}$. Without loss of generality, we assume $x_{0} \preceq y_{0}$. In this case, the sequence $\left\{x_{n}\right\}$ is monotone increasing, i.e., $x_{n} \preceq x_{n+1}$, for any $n \in \mathbb{N}$. Let $z_{1}$ and $z_{2}$ be two $\tau$-cluster points of $\left\{x_{n}\right\}$. Fix $k \in \mathbb{N}$. Since $\left\{x_{n}\right\}$ is monotone increasing and the order interval $\left[x_{k}, \rightarrow\right)$ is $\tau$-closed, we conclude that $z_{i} \in\left[x_{k}, \rightarrow\right)$, for $i=1,2$. Hence $\left\{x_{n}\right\} \subset\left(\leftarrow, z_{i}\right]$, for $i \in\{1,2\}$, holds which implies for the same reason $z_{j} \in\left(\leftarrow, z_{i}\right]$, for $i, j \in\{1,2\}$. Hence $z_{1}=z_{2}$, which implies that $\left\{x_{n}\right\}$ has at most one $\tau$-cluster point.

Note that if we assume $C$ is $\tau$-compact, then $\left\{x_{n}\right\}$ is $\tau$-convergent to some $z \in C$ and either $x_{n} \preceq z$ or $z \preceq x_{n}$, for any $n \in \mathbb{N}$. Before, we state the main result of this section, define $\mathcal{K}(C)$ to be the set of all nonempty compact subsets of $C$. 
Theorem 3.5. Let $(X,\|\|,. \preceq)$ and $\tau$ be as described above. Assume $X$ satisfies the monotone $\tau$-Opial condition. Let $\mathrm{C}$ be a $\tau$-compact, convex and bounded nonempty subset of $\mathrm{X}$ not reduced to one point. Let $\mathrm{T}: \mathrm{C} \rightarrow \mathcal{K}(\mathrm{C})$ be a monotone nonexpansive multivalued mapping with nonempty values. Assume $\mathrm{C}_{\mathrm{T}}$ is not empty. Then $\mathrm{T}$ has a fixed point.

Proof. Since $\mathrm{C}_{\mathrm{T}}$ is not empty, pick $\mathrm{x}_{0} \in \mathrm{C}_{\mathrm{T}}$. Fix $\lambda \in(0,1)$. Without loss of generality, assume there exists $y_{0} \in \mathrm{T}\left(x_{0}\right)$ such that $x_{0} \preceq y_{0}$. Consider the sequences $\left\{x_{n}\right\}$ and $\left\{y_{n}\right\}$ in $C$ generated by $\lambda, x_{0}$ and the iteration (3.1). Since $C$ is $\tau$-compact, we know that $\left\{x_{n}\right\}$ is $\tau$-convergent to some point $z \in C$. Since $\left\{x_{n}\right\}$ is monotone increasing, then we have $x_{n} \preceq z$, for all $n \in \mathbb{N}$. Since $T$ is monotone multivalued nonexpansive and $y_{n} \in T\left(x_{n}\right)$, there exists $z_{n} \in T(z)$ such that $y_{n} \preceq z_{n}$ and

$$
\left\|y_{n}-z_{n}\right\| \leqslant\left\|x_{n}-z\right\|, \quad \forall n \in \mathbb{N} .
$$

Since $T(z)$ is compact, there exists a subsequence $\left\{z_{\phi(n)}\right\}$ of $\left\{z_{n}\right\}$ such that $\left\{z_{\phi(n)}\right\}$ converges to $v \in T(z)$. Moreover, we have $\lim _{n \rightarrow+\infty}\left\|x_{n}-y_{n}\right\|=0$, since $C$ is bounded. Hence, we have

$$
\limsup _{n \rightarrow \infty}\left\|y_{\phi(n)}-z_{\phi(n)}\right\|=\limsup _{n \rightarrow \infty}\left\|y_{\phi(n)}-v\right\|=\limsup _{n \rightarrow \infty}\left\|x_{\phi(n)}-v\right\|
$$

which implies

$$
\limsup _{n \rightarrow \infty}\left\|x_{\phi(n)}-v\right\| \leqslant \limsup _{n \rightarrow \infty}\left\|x_{\phi(n)}-z\right\| .
$$

Let us prove that $z \preceq v$. Indeed, we have $x_{n} \preceq y_{n} \preceq z_{n}$, for any $n \in \mathbb{N}$. Since $\left\{x_{n}\right\}$ is increasing and the order intervals are closed, we conclude that $x_{n} \preceq v$. Finally since $\left\{x_{n}\right\} \tau$-converges to $z$ and the order intervals are $\tau$-closed, we obtain $z \preceq v$. Since $X$ satisfies the monotone $\tau$-Opial condition, we conclude that $z=v \in \mathrm{T}(z)$, i.e., $z$ is a fixed point of $\mathrm{T}$.

\section{Acknowledgment}

This work was supported by the Deanship of Scientific Research (DSR), King Abdulaziz University, Jeddah, under Grant No. (363-10-D1438). The authors, therefore, gratefully acknowledge the DSR technical and financial support.

\section{References}

[1] M. R. Alfuraidan, M. A. Khamsi, A fixed point theorem for monotone asymptotically nonexpansive mappings, Proc. Amer. Math. Soc., (to appear). 2.2, 2

[2] M. Bachar, M. A. Khamsi, Recent contributions to fixed point theory of monotone mappings, J. Fixed Point Theory Appl., 19 (2017), 1953-1976. 1

[3] S. Banach, Sur les opérations dans les ensembles abstraits et leur application aux équations intégrales, Fund. Math., 3 (1922), 133-181. 1

[4] T. D. Benavides, P. L. Ramírez, Fixed-point theorems for multivalued non-expansive mappings without uniform convexity, Abstr. Appl. Anal., 2003 (2003), 375-386. 1

[5] F. E. Browder, Nonlinear operators and nonlinear equations of evolution in Banach spaces, Nonlinear functional analysis (Proc. Sympos. Pure Math., Vol. XVIII, Part 2, Chicago, Ill., 1968), Amer. Math. Soc., Providence, R. I., (1976), 1-308. 1

[6] T. Domínguez Benavides, J. García Falsett, M. A. Japón Pineda, The $\tau$-fixed point property for nonexpansive mappings, Abstr. Appl. Anal., 3 (1998), 343-362. 2, 2

[7] R. M. Dudley, On sequential convergence, Trans. Amer. Math. Soc., 112 (1964), 483-507. 3

[8] K. Goebel, W. A. Kirk, Iteration processes for nonexpansive mappings, Topological methods in nonlinear functional analysis, Toronto, Ont., (1982), Contemp. Math., Amer. Math. Soc., Providence, RI, 21 (1983), 115-123. 3.3

[9] K. Goebel, W. A. Kirk, Topics in metric fixed point theory, Cambridge Studies in Advanced Mathematics, 28. Cambridge University Press, Cambridge, (1990). 3.3

[10] S. Ishikawa, Fixed points and iteration of a nonexpansive mapping in a Banach space, Proc. Amer. Math. Soc., 59 (1976), 65-71. 3 
[11] M. A. Khamsi, On uniform Opial condition and uniform Kadec-Klee property in Banach and metric spaces, Nonlinear Anal., 10 (1996), 1733-1748. 2

[12] M. A. Khamsi, W. A. Kirk, An introduction to metric spaces and fixed point theory, Pure and Applied Mathematics (New York), Wiley-Interscience, New York, (2001). 1

[13] M. A. Krasnosel'skiǔ, Two remarks on the method of successive approximations, (Russian) Uspehi Mat. Nauk (N.S.), 10 (1955), 123-127. 3

[14] E. Lami Dozo, Multivalued nonexpansive mappings and Opial's condition, Proc. Amer. Math. Soc., 38 (1973), $286-292$. $1,2,2$

[15] T.C. Lim, Asymptotic centers and nonexpansive mappings in conjugate Banach spaces, Pacific J. Math., 90 (1980), 135143. 2

[16] J. T. Markin, A fixed point theorem for set valued mappings, Bull. Amer. Math. Soc., 74 (1968), 639-640. 1

[17] Z. Opial, Weak convergence of the sequence of successive approximations for nonexpansive mappings, Bull. Amer. Math. Soc., 73 (1967), 591-597. 2, 2

[18] A. C. M. Ran, M. C. B. Reurings, A fixed point theorem in partially ordered sets and some applications to matrix equations, Proc. Amer. Math. Soc., 132 (2004), 1435-1443. 1 